\title{
Development of drone-borne aerial calibration pulser system for radio observatories of ultra-high energy air showers
}

\author{
J. Nam, ${ }^{1,2}$ C.-Y. Kuo, ${ }^{1,2, *}$ B.K. Shin, ${ }^{3}$ P. Chen, ${ }^{1,2}$ Y. Chen, ${ }^{1,2}$ S.-Y. Hsu, ${ }^{1}$ J.-J. Huang, ${ }^{1,2}$ \\ M.-H. A. Huang, ${ }^{4}$ C.H. Leung, ${ }^{1,2}$ T.-C. Liu, ${ }^{1,2}$ S.-H. Wang, ${ }^{1,2}$ M.-Z. Wang, ${ }^{1,2}$ Y.-H. \\ Wang, ${ }^{1,2}$ D. Besson, ${ }^{5,7}$ A. Novikov, ${ }^{5,6}$ C. Hornhuber ${ }^{5,7}$ and R. Young ${ }^{5,6}$ \\ ${ }^{1}$ Department of Physics, National Taiwan University, Taipei, Taiwan. \\ ${ }^{2}$ Leung Center for Cosmology and Particle Astrophysics, National Taiwan University, Taipei, Taiwan. \\ ${ }^{3}$ Ulsan National Institute of Science and Technology, Ulsan, Korea. \\ ${ }^{4}$ Department of Energy and Resources, National United University, Miaoli, Taiwan \\ ${ }^{5}$ Department of Physics and Astronomy, University of Kansas, Lawrence, USA. \\ ${ }^{6}$ Instrumentation Design Lab, University of Kansas, Lawrence, USA. \\ ${ }^{7}$ National Research Nuclear University MEPhI (Moscow Engineering Physics Institute), Moscow, Russia \\ E-mail: namjiwoo@gmail.com, r06222016@ntu.edu.tw
}

\begin{abstract}
We present a portable calibration pulser system applied to radio antennas in order to detect ultrahigh energy cosmic rays and neutrinos. The system consists of a solid-state high-voltage impulse generator, a digital attenuator, a wide band (150-350 MHz) bi-cone antenna, and a differential GPS. Taking an advantage of light weight of each component (less than $1.4 \mathrm{~kg}$ ), we developed an airborne calibration system by attaching them on a commercial drone. This system will be used for TAROGE experiment in high mountains as well as in Antarctica. We will report on its design, construction, performance, and potential applications for future radio experiments.
\end{abstract}

$37^{\text {th }}$ International Cosmic Ray Conference (ICRC 2021)

July 12th-23rd, 2021

Online - Berlin, Germany

\footnotetext{
*Presenter
} 


\section{Introduction}

In recent years, radio technique has been regarded as a promising method to detect ultra-high energy cosmic rays and cosmic neutrinos [1]. Air shower initiated by neutrino induces coherent emission from the geo-magnetic radiation and Askaryan radiation. Antenna array can receive such radio impulse from several tens of kilometer away so that a single radio station can provide a large effective area comparing to conventional particle detectors. Several radio experiments have proved the potential of radio technique, like ANITA, ARA and TAROGE [2, 3]. Besides, radio detector can provide high duty cycle and can be deployed faster with less effort. Therefore, experiments based on radio method progress rapidly in these decades.

One of the most important data analysis methods in the radio experiments is interferometry technique which can provide arrival directions of radio pulses [4]. A precise calibration, especially timing calibration, is a crucial experimental step for the interferometry technique. Conventionally, ground pulser, balloon-borne pulser, or bright cosmic sources are used as calibration references [5, 6]. However, sky coverage for those methods are limited. Multiple calibration stations would be deployed, which would not be practical solution for some experiments such as TAROGE. We have developed a drone-borne cal-pulser that can be applied to various radio observatories. Similar ideas have been presented by AERA, TA and Bleien Observatory [5, 7-9]. However, our system is the most advanced using a solid-state impulse generator and differential GPS (D-GPS) technique, which provides a compact, light-weighted, portable, and accurate position information. In this paper, we will show the design, development and performance in applications to existing TAROGE experiment.

\section{Design, development and performance}

The cal-pulser system consists of a pulse transmitter and a D-GPS system within an RF shielding box. The system diagram is shown in the Figure 1. The pulse transmitter generates impulsive signals with controlled amplitude while D-GPS records the position of the drone with an accuracy of several centimeter. The system is compact and light-weighted. Total weight of the system is around 1.4 $\mathrm{kg}$, which is able to be attached on a commercial drone - DJI M600Pro, as shown in Figure 2. This drone can provide a flight time around 25 minutes for each calibration. Typical distance between drone and antenna array during the calibration is around $600 \mathrm{~m}$ by concerning the RFI of the drone, resolution of the D-GPS and flight time of the drone. At this distance, the angular uncertainty of the pulser with respect to the antenna array is about $0.01^{\circ}$, which is sufficiently small for the calibration of antenna array with sub-degree resolution. Besides, the drone can be pre-programmed with way-points to perform automatic grid scan in entire field of view $\left(-50^{\circ} \sim 50^{\circ}\right.$ in azimuth and $-7^{\circ} \sim 7^{\circ}$ in elevation) with $5^{\circ}$ grid spacing in azimuth with two sets of batteries. In the following section, we will present the detailed implementation of the transmitter, the performance of D-GPS and the measurement of RFI. A brief introduction of the antenna array in TAROGE experiment to be calibrated is covered in the last section. 


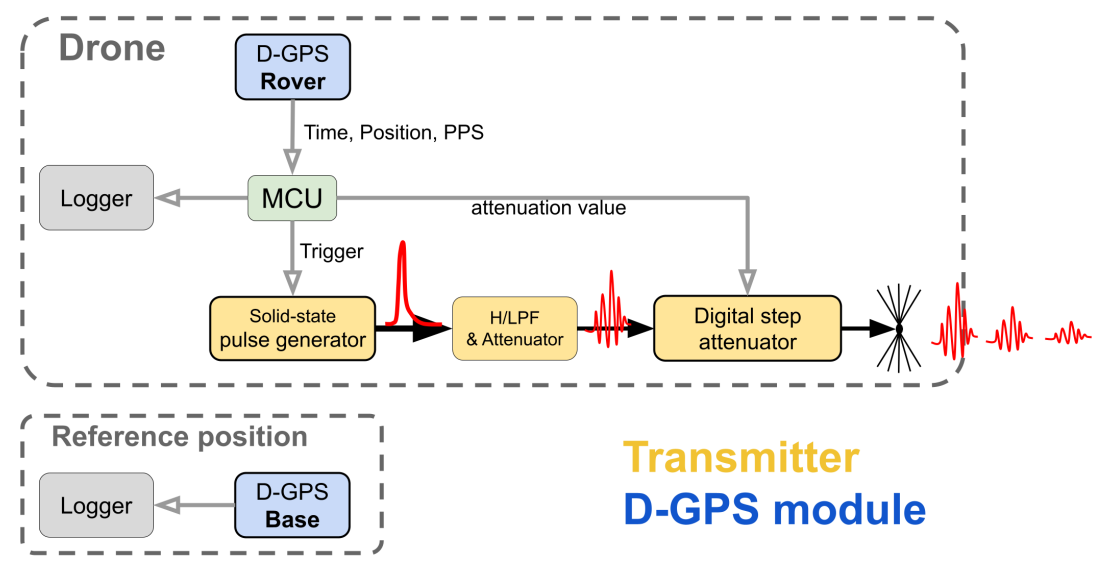

Figure 1: System diagram of the drone-borne cal-pulser system.

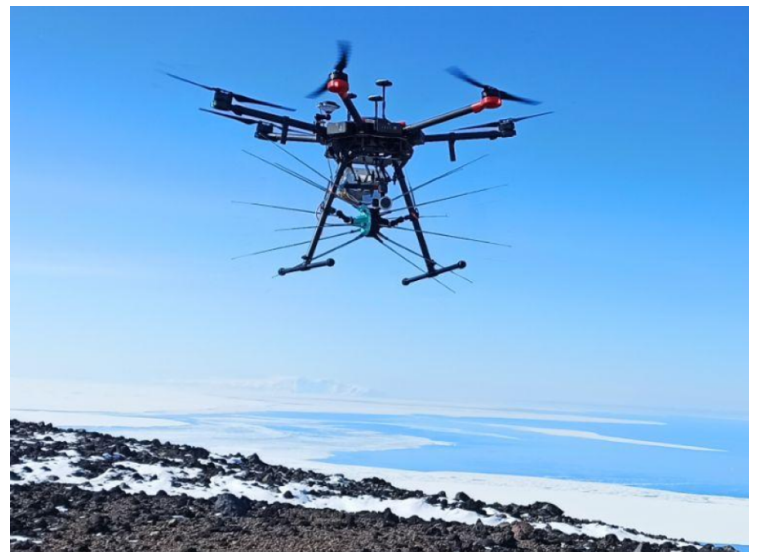

Figure 2: Drone-borne cal-pulser during the calibration of TAROGE-M system in Antarctica

\subsection{Pulse transmitter}

The pulse transmitter can generate high power impulsive signal with pre-programmed stepped amplitude at accurate repetition rate and time. Clock of micro-controller is synchronized with PPS signal so that it can trigger the pulse source in a repetition rate from 1 to $40 \mathrm{~Hz}$ with a timing uncertainty of $0.3 \mu \mathrm{s}$, which allow us to directly identify the calibration signal by applying a constrain on trigger timestamp of antenna system. Impulsive signals are produced by an avalanche pulse source which provides stable uni-polar pulse with a peak amplitude at around $1.5 \mathrm{~V}$ and a RMS of peak voltage at $36 \mathrm{mV}$ (measured with additional $-42 \mathrm{~dB}$ attenuator), as shown in Figure 3a. To prevent damaging on the following devices, a $-13 \mathrm{~dB}$ attenuator reduces the power of the pulses and two on-board filters limit the bandwidth from $115 \mathrm{MHz}$ to $1200 \mathrm{MHz}$. The resulting spectrum is shown in the Figure 3b. Then amplitude of the impulse is adjusted by a digital attenuator. Finally, the impulses are transmitted via a broadband bi-cone antenna with a bandwidth from $180 \mathrm{MHz}$ to $350 \mathrm{MHz}$. The waveform and spectrum of transmitted pulses are shown in Figure 4 and Figure 5a. The antenna has a well-defined polarization with a cross polarization leakage lower than $-15 \mathrm{~dB}$, as shown in Figure 5b, so that we can calibrate the response of each polarization of antenna array respectively. 


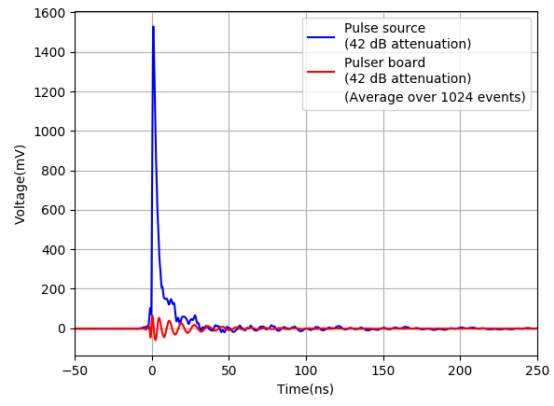

(a) Waveform.

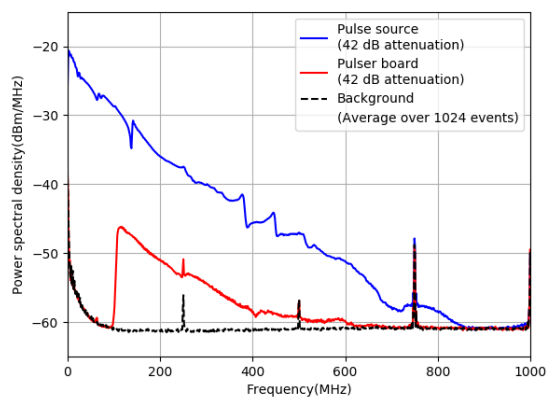

(b) Spectrum.

Figure 3: Waveform and spectrum of impulsive signal from the avalanche pulse source (Pulse source) and the signal passed through on-board attenuator and filter (Pulser board).

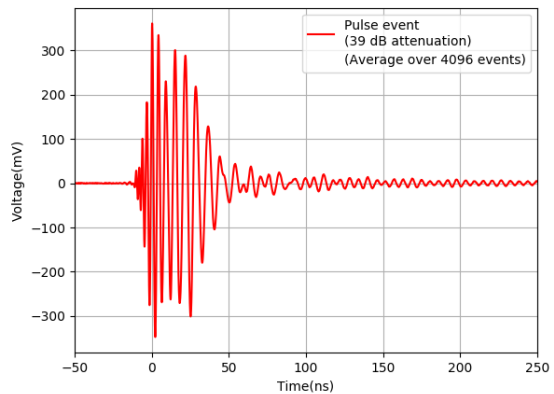

(a) Waveform

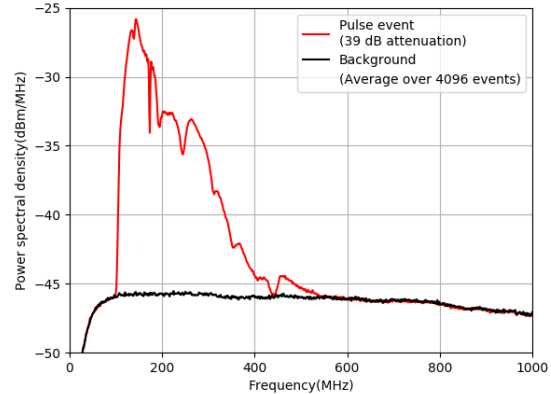

(b) Spectrum.

Figure 4: Waveform and spectrum of impulsive signals transmitted via bi-cone antenna measured with antenna and LNA used in TAROGE experiment[12]

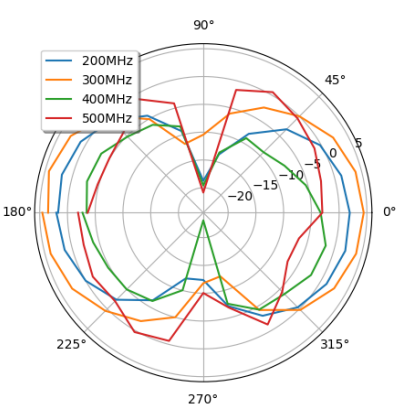

(a) Beam pattern of the bi-cone antenna.

The beam-width is around 60 degree.

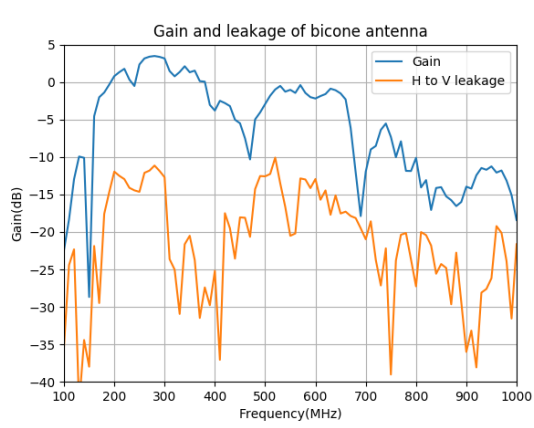

(b) The gain and cross-pol leakage of the bi-cone antenna.

Figure 5: Characteristic of broadband bi-cone antenna. 


\subsection{Differential GPS}

A differential GPS based on the carrier-phase measurement is applied to provide position of the transmitter with an accuracy of several centimeter, while conventional GPS only provide an accuracy of $3 \mathrm{~m}$ in horizontal and $10 \mathrm{~m}$ in vertical. D-GPS system consists of two GPS receivers - base unit and rover unit. Base unit is placed at a known reference position, while rover unit, together with the transmitter, is placed on the drone. Two units record the GPS measurement simultaneously. Based on the known reference position, base unit can provide a correction information of ionsphere interference to rover unit. The correction is done in an offline processing, with RTK-LIB software[10], to reduce effort on deployment in the field and the possible RFI from the radio link between two units.

The accuracy of D-GPS is measured to be within several centimeter on the ground in the static and kinematic mode. A comparison between distance measurement by GPS and D-GPS is shown in Figure 6. However, the accuracy of this system in the air is not precisely measured due to limitation of accessibility. Alternatively, we measure the position of the drone by the antenna array of TAROGE experiment. The interferometry technique can provide high resolution up to sub-degree level in the high elevation angle due to less interference from ground reflection. The expected position by the D-GPS and reconstructed position by interferometry method are consistent up to sub-degree level, which will be showed in the next section. From these results, we show that the position uncertainty of D-GPS is suitable for the calibration for antenna array with sub-degree resolution.

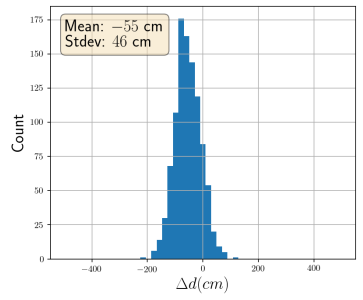

(a) GPS

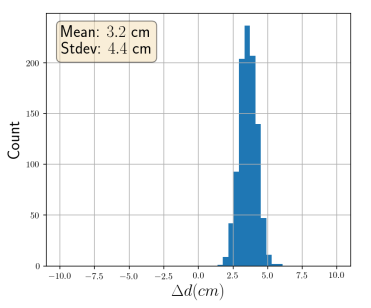

(b) D-GPS in kinematic mode

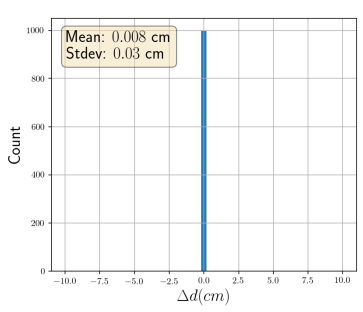

(c) D-GPS in static mode

Figure 6: Comparison between distance measurement based on GPS and D-GPS. The true distance between base and rover is $12.58 \mathrm{~m}$, measured by theodolite and photogrammetry.

\subsection{RFI measurement and RF shielding}

Low RFI from cal-pulser system and drone are important to ensure the calibration without noise interference and to reduce fake trigger. Therefore, we conduct an RFI measurement for drone and cal-pulser system in the anechoic chamber with LNA and log periodic dipole antenna (LPDA) used in TAROGE experiments[2]. The test components are placed $5 \mathrm{~m}$ in front of the LPDA with additional attenuator to prevent the signal from exceeding the dynamic range of the amplifier. The RFI of the drone composes of impulsive noise and continuous wave $(\mathrm{CW})$ noise. These noise are negligible at an attenuation of $-30 \mathrm{~dB}$, corresponding to $150 \mathrm{~m}$ away from the antenna. Likewise, strong impulsive noise is also emitted from the pulser system. The impulsive noise is too strong 
to be neglected. Therefore, we utilized a light-weighted stainless shielding box, which can provide more than $-40 \mathrm{~dB}$ power attenuation, to suppress such noise.

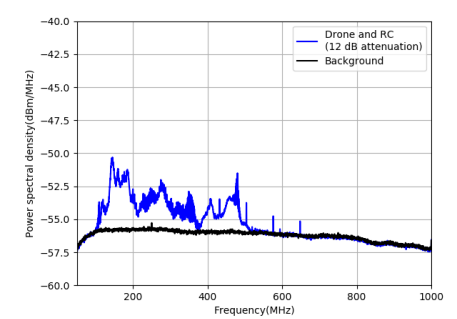

(a) Drone and the controller (RC).

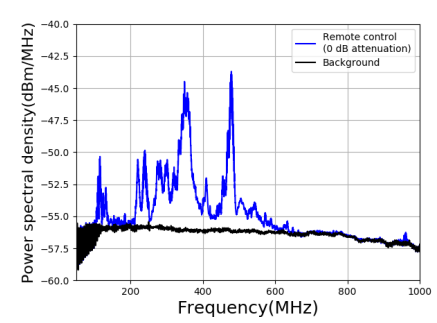

(b) Remote controller (RC).

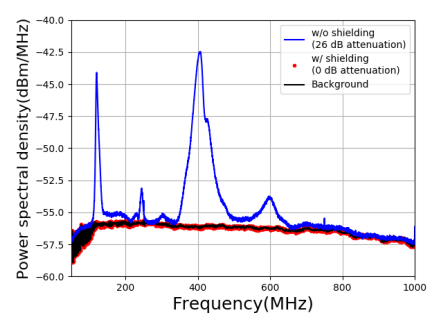

(c) Pulser system.

Figure 7: RFI measurement in the anechoic chamber.

\subsection{Test with TAROGE experiment}

Taiwan Astro-particle Radio-wave Observatory for Geo-synchrotron Emission (TAROGE) are radio observatories on the high mountains of Taiwan's east coast working in $180 \mathrm{MHz}-350 \mathrm{MHz}$ frequency band[2, 12], as well as the high mountains in Antarctica working in $150 \mathrm{MHz}-500 \mathrm{MHz}$ frequency band. They are designed to detect the near-horizontal air showers induced by the cosmic rays and the earth-skimming tau neutrinos. The station consists of several LPDAs pointing horizontally in vertical ( Vpol) and horizontal ( $\mathrm{Hpol})$ polarization. The antennas are placed with a horizontal spacing of $7 \mathrm{~m}$ and a vertical spacing of $5 \mathrm{~m}$. The target resolution of the system is at sub-degree level in the near horizontal direction to distinguish the interesting up-going events from down-going events.

\section{Result and discussion}

This drone-borne cal-pulser has been applied to the calibration of TAROGE-2,3,4 in Taiwan and TAROGE-M in Antarctica. To verify the pulser is properly working in the air, we utilized the instruments of TAROGE experiment to validate the function of cal-pulser. We select the data from the bore-sight of both transmitter and antenna array so that the effect of antenna response can be minimized. Besides, for the verification of the drone position, we follow the best event selection showed in [13] by using events from high elevation angle and excluding events in the biased region to reduce the effect of ground reflection.

\subsection{Function of transmitter}

Amplitude control, timing of the pulser are verified by a lab measurement and the calibration of TAROGE-4 in 2019. Figure 8a shows the trigger timestamp of first pulses in a second. The stable timestamp indicates the clock on the micro-controller is well-synchronized with the clock in the antenna array via GPS. Figure 8b shows the attenuation set on the transmitter and the transmitting power of pulser event estimated by antenna array of TAROGE-4. The inverse linear relationship shows the amplitude controller is properly functioned. 


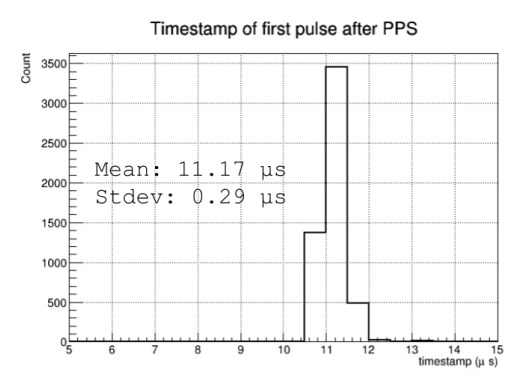

(a) Trigger timestamp measured in the lab with ay versus attenuat set TAROGE trigger system. array versus attenuation set on the pulser. The slope

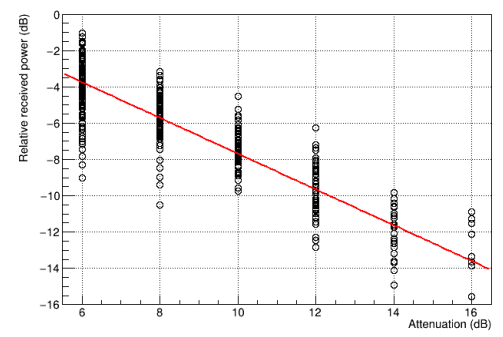

(b) Relative transmitted power estimated by antenna of fit line is -0.99 , which is indicated the amplitude controller is functioning properly in the air

Figure 8: Measurement of pulser events by TAROGE-4

\subsection{Drone position}

As noted in previous section, we verify the in-air drone position by interferometry method. Without huge interference from ground reflection, the reconstructed arrival direction of the pulser events can reach sub-degree resolution. Figure 9 show the difference between reconstructed arrival direction by interferometry and the expected value by D-GPS from the calibration of TAROGE-M in 2020. Two position measurements are well-matched within $0.2^{\circ}$ in azimuth angle and $0.3^{\circ}$ in elevation angle, which are around the targeted resolution of antenna array.

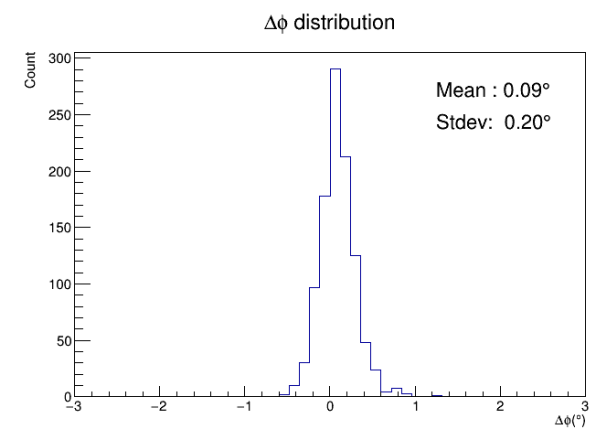

(a) Azimuth angle

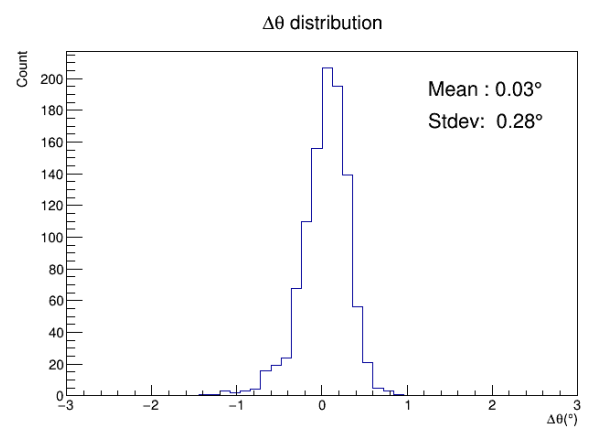

(b) Elevation angle

Figure 9: The difference between reconstructed arrival direction by interferometry and the expected value by D-GPS.

The result shows the uncertainty of the drone position, measured by D-GPS, can't be identified by antenna array due to sub-degree resolution of antenna array. However, it also indicates that the uncertainty of drone position is small enough for the calibration of the antenna array with sub-degree resolution. Further verification of drone position based on photogrammetry method will be conducted soon to provide accurate estimation of the position uncertainty in the air. 


\section{Conclusion}

With the state-of-the-art impulsive source and D-GPS technique, we show that the drone-borne cal-pulser is successfully developed and tested. It makes us easier to do calibration in the field and provide us a practical method to investigate the response of antenna array from any direction of interest. Besides, further application to model ground reflection of antenna array with drone has been investigated. With these advantages, drone-borne calibration method is very promising for the radio detection of air shower.

\section{Acknowledgement}

We would like to thank the support from Taiwan Minister Of Science and Technology (MOST) and from Korea Polar Research Institute (KOPRI).

\section{References}

[1] F. Schröder, Prog. Part. Nucl. Phys. 93 (2017) 1.

[2] J. Nam et al., Int. J. Mod. Phys. D 25 (2016) 1645013.

[3] P. Gorham et al., Phys. Rev. Lett. 126 (2021) 071103.

[4] A. Romero-Wolf et al., Astropar. Phys. 60 (2015) 72.

[5] C. Chang et al., Publ. Astron. Soc. Pac 127 (2015) 957, 1131.

[6] P. Gorham et al., Nucl. Instrum. Meth. A918 (2019) 60.

[7] B. Florian, EPJ Web Conf. 135 (2017) 01014.

[8] A. Aab et al., JINST 12 (2017) T10005.

[9] M. Hayashi et al., EPJ web conf. 210 (2019) 05015.

[10] T., Tomoji et al., IS-GNSS 1(2009).

[11] J. Nam et al., PoS(ICRC2019)967.

[12] T.-C., Liu, PoS(ICRC2017)234.

[13] S.H. Wang, PoS(ICRC2021)1173 . 